\title{
Experimental measurements of gravity casting flow
}

\author{
Libor Lobovský ${ }^{1, *}$ and Tomáš Mandys ${ }^{1}$ \\ ${ }^{1}$ NTIS - New Technologies for the Information Society, Faculty of Applied Sciences, University of West Bohemia, Univerzitní 8,30100 \\ Plzeň, Czech Republic
}

\begin{abstract}
An experimental investigation of casting processes performed under well controlled laboratory conditions is presented. Casting molds made of transparent material allow for analysis of flow patterns by means of standard optical measurement methods. The tests are carried out for selected highly-viscous Newtonian fluids at room temperature. The physical properties of the test fluids (i.e. viscosity, surface tension and density) are identified. As a result, an extensive set of experimental data is provided.
\end{abstract}

\section{Introduction}

Manufacturing processes that involve casting of liquid material driven by a body force (e.g. gravity, centrifugal force) are well employed in a number of industrial applications such as rapid prototyping, medical device molding, replacement part manufacturing etc. The castings can be made of any material that solidifies due to either thermal change (metals, some plastics) or due to chemical processes that happen after mixing several components together (plaster, clay or resins).

The casting mold geometry plus a location and a number of inlets and outlets are crucial parameters which have an impact on casting quality and its costs. These geometric properties are vital especially during casting of complex thin-walled three-dimensional structures. In order to optimise the design of the casting mold, understanding and prediction of the casting flow is essential. This can be achieved empirically or by computational and experimental modelling.

In situ experimental measurements of casting processes are challenging especially due to the complexity of mold geometry and due to the character of the cast material which is often liquified at high temperatures. Nevertheless, several publications on in situ measurements using $\mathrm{X}$-ray radiography can be found e.g. in [1,2]. However, the casting process can be well approximated using a water analogue model $[3,4]$ which enables a good control over the model parameters in laboratory conditions.

This study focuses on experimental investigation of the free surface flow inside a rigid mold during gravity casting. Thermodynamic and chemical effects are neglected and an analogue approach is applied.

\section{Experimental setup}

In the following, highly viscous Newtonian fluids are used as an analogue to the cast material and the geometry of the

*e-mail: lobo@kme.zcu.cz molds is made of a rigid transparent material (polymethyl methacrylate) so that the evolution of the gravity driven free surface flow can be assessed by means of standard optical measurement methods.

Two elementary experimental setups are considered, casting flow in a long horizontal channel, Figure 1, and in a complex vertical labyrinth, Figure 2 . A special attention is paid to the kinematics of propagation of the cast material and to the shape of the free surface profile. All experiments are performed at standard room temperature.

\subsection{Horizontal channel}

The first experimental setup is designed so that it enables analysis of propagation of the cast material in a mold of simple geometry with a single inlet and a single outlet. The setup geometry consists of two connected parts, a vertical reservoir and a horizontal mold, as depicted in Figure 3. The horizontal mold is a $1160 \mathrm{~mm}$ long horizontal channel that winds in a spiral. The channel crosssection is rectangular and does not vary along the channel length, the channel height is $3 \mathrm{~mm}$ and its width is $10 \mathrm{~mm}$. The length of the horizontal channel is large in comparison to its cross-sectional dimensions. Thus the physical effects get pronounced as the cast material propagates along the channel length and a level of the material in the vertical reservoir decreases.

Initially, the vertical reservoir holds $90 \mathrm{ml}$ of liquid. Before the experiment is started, the test liquid is held inside the reservoir by a removable gate which obstructs the horizontal channel. Thus, the horizontal channel is kept empty and dry. At the beginning of each measurement, the gate is removed abrubtly in the vertical direction and the liquid flows into the rigid channel solely under gravity. A free flow of air is assured by a cylindrical opening at the other end of the horizontal channel.

The spiral shape of the experimental channel simplifies the optical measurements as the channel fits well within 


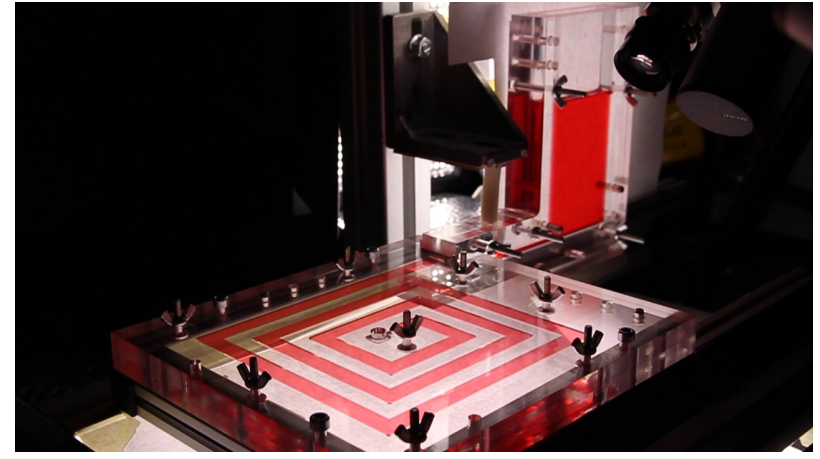

Fig. 1. Experimental setup for the tests of gravity casting into the horizontal channel.

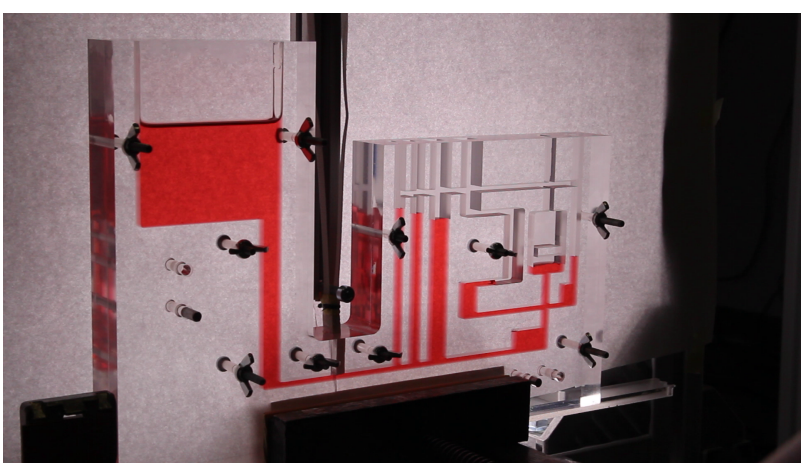

Fig. 2. Experimental setup for the tests of gravity casting into the vertical labyrinth.

a camera field of view. Thus all parts of the channel may be examined at relatively high resolution when the entire channel spiral is viewed from the top.

\subsection{Vertical labyrinth}

The second experimental setup provides a rather complex vertically oriented geometry which enables analysis of gravity casting into a labyrinth of channels of various sizes and shapes as displayed in Figure 4. All labyrinth channels have a rectangular cross-section while the entire labyrinth has a constant breadth of $10 \mathrm{~mm}$.

Next to the labyrinth of vertical channels, there is a reservoir of cast material which is connected to the labyrinth via a $3 \mathrm{~mm}$ high horizontal channel. This channel is the only inlet into the vertical labyrinth and prior to each experiment it is obstructed by a removable gate. The reservoir initially holds $72 \mathrm{ml}$ of liquid, while the labyrinth behind the gate is kept empty and dry. After an abrubt gate removal, the cast material is free to flow (driven solely by gravity) into the labyrinth channels. First, the cast material reaches three straight vertical channels with thickness of 3,5 and $10 \mathrm{~mm}$. The horizontal channel continues and its end opens to a $23 \times 13 \times$ $10 \mathrm{~mm}$ large chamber. The distal part of the chamber is an origin of a trident of vertical channels that vary in size and shape, see Figure 4.

All six vertical channels are designed so that they end at the top edge of the vertical labyrinth mold. Thus they

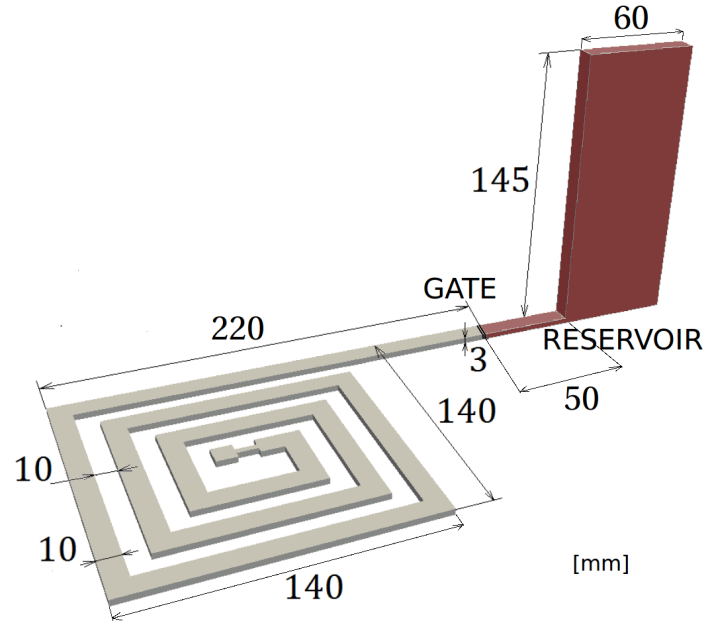

Fig. 3. Horizontal channel: setup geometry and its dimensions.

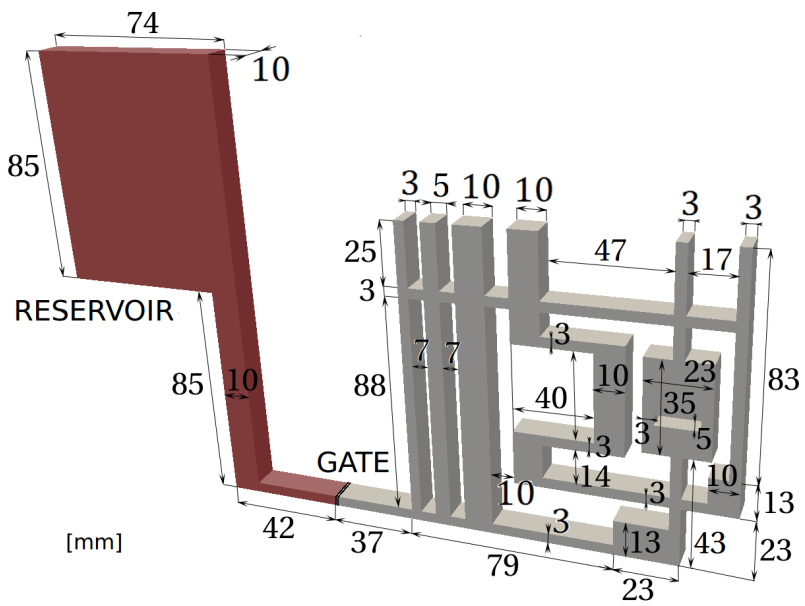

Fig. 4. Vertical labyrinth: setup geometry and its dimensions.

form natural outlets which enable a free flow of air out of the labyrinth. In addition, $25 \mathrm{~mm}$ below the top edge of the mold there is a $3 \mathrm{~mm}$ high horizontal channel which connects all the vertical ones.

\subsection{Test fluids}

Both experimental setups (described above) are used for testing the gravity casting flow of in-house prepared highly-viscous fluids. A fresh batch of coloured aqueous sugar solution is prepared for each of the two setups. Since each batch is produced separately, the test fluid for the horizontal channel tests has slightly different physical properties than the fluid for the vertical labyrinth tests.

Material properties (i.e. viscosity, surface tension and density) of the test fluids are identified experimentally and described in section 3.1 below. In order to assess the dynamic viscosity, a rotational rheometer with coaxial cylinders geometry is applied. The surface tension is measured using a force tensiometer with Wilhelmy plate. Laboratory weighing scales and precise pipetting is utilised while assesing the fluid density. The measurements are performed 


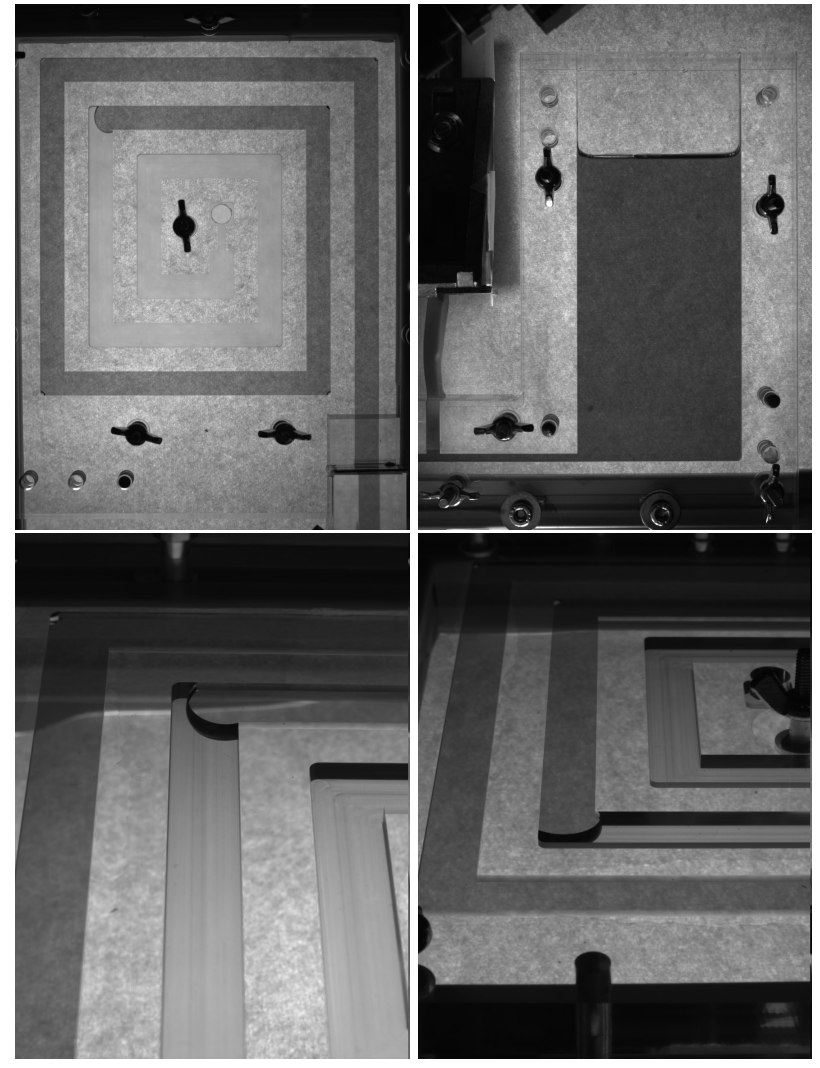

Fig. 5. Horizontal channel: field of view of the four cameras capturing a single time instant of the casting flow experiment.

both for fresh samples and for the used samples right after their application in the gravity casting experiments.

\subsection{Data acquisition}

The gravity casting experiments are analysed based on optical measurements by a system of four synchronised cameras, Figure 5 and Figure 6. In each experiment, two cameras cover the entire test scene and two cameras are focused on details.

The camera setup is designed as follows. The camera no. 1 records propagation of the test fluid through the entire mold geometry behind the gate. The camera no. 2 captures the evolution of the fluid free surface in the reservoir during the experiment. The cameras no. 3 and no. 4 focus on a selected region of the mold geometry in detail from two angles so that an information about three-dimensional shape of the fluid front can be assessed.

The framerate of $20 \mathrm{~Hz}$ is applied for all the cameras so that a sufficient time resolution is provided to capture the initial phase of the gravity flow after the gate removal. All cameras have an image resolution of $2336 \times 1752 \mathrm{px}$.

\section{Results and discussion}

During the execution of the experimental tests, major attention is paid to kinematics of the gravity driven flow for both the horizontal channel as well as the vertical

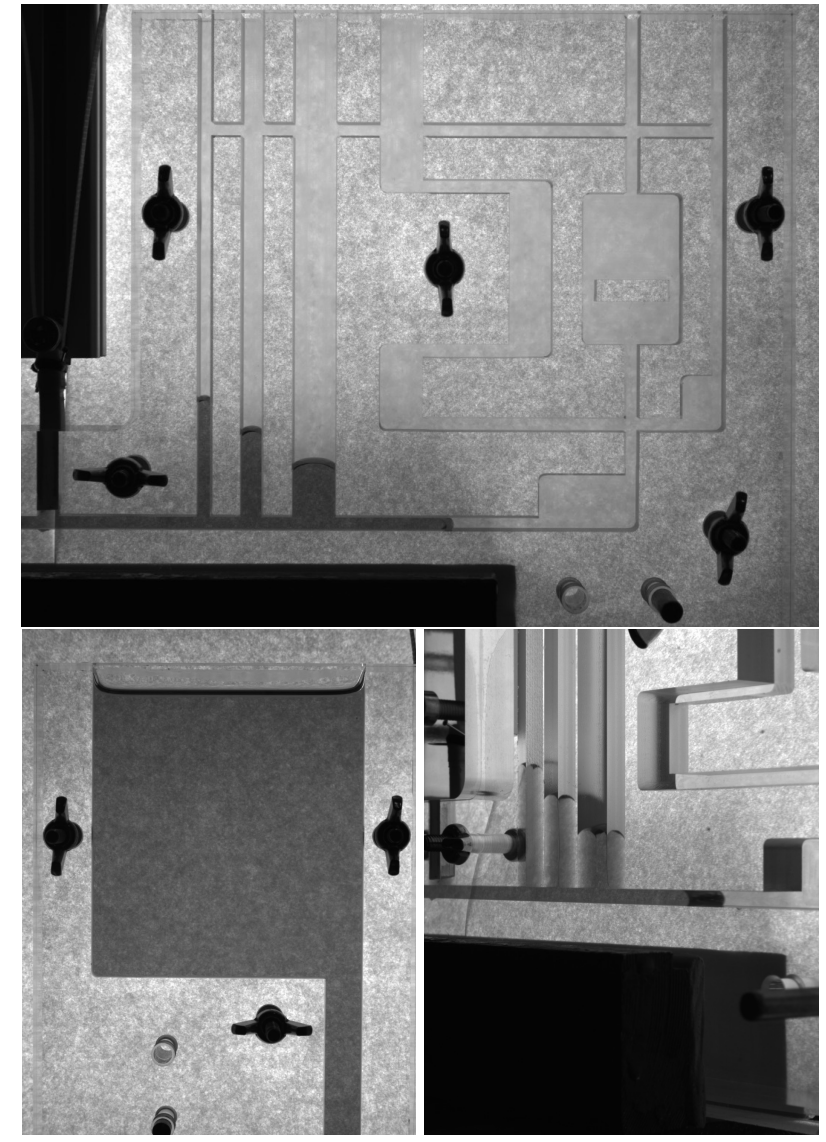

Fig. 6. Vertical labyrinth: field of view of the first three (out of four) cameras at a single time instant of the casting experiment.

labyrinth. Especially the propagation of the fluid front through the mold geometry is of interest.

In order to examine the repeatibility of the tests, a serie of 10 runs is executed for each experimental setup. Each test run is performed for a fresh well stirred sample of the test fluid that is casted into the well cleared and dried casting mold. In addition, every run of gravity casting tests is accompanied with identification of material properties of the currently used fluid sample.

\subsection{Rheological measurements}

Measurements of the viscosity and of the surface tension are performed for both the fresh sample before and the used sample after execution of the casting flow experiments. The density measurements are performed for both fresh and used samples in the very end of each experimental campaign.

The key factor influencing propagation of the casted fluid inside the mold geometry is fluid's dynamic viscosity. The rheological measurements are performed for a wide range of shear rates using a rotational rheometer with coaxial cylinders, i.e. these measurements are driven by a prescribed rotational speed of the internal cylinder while the external cylinder is static. The shear stress in the fluid is assessed based on the torque exerted on the internal cylinder. 
Basically two elementary test scenarios are applied. First the dynamic viscosity is tested for a continuously changing (either linearly increasing or lineraly decreasing) value of the shear rate (linear runup/rundown test). Second the step-wise function of the shear rate is defined so that a steady response of the fluid to several prescribed shear rate values can be assessed. A serie of overall 42,33 respectively, measurements of the dynamic viscosity of the test fluid is executed during the experimental campaign for the horizontal channel, the vertical labyrinth respectively. Although rather lower shear rates are expected in the gravity casting tests described below, the response of the test fluid to shear rates in a range of $\langle 0 ; 1945\rangle \mathrm{s}^{-1}$ is examined.

Results of all rheological measurements of the test fluid used in the horizontal channel campaign are shown in Figure 7. The figure is divided into three colour-coded ranges of shear rate values. The green colour is used for an optimal measurement range of shear rates (based on the specifications of the rotational rheometer and the applied coaxial cylinder geometry). The yellow colour denotes limits of applicable measurement range. The red colour region is out of the applicable measurement range. It can be observed that the measured curves are smooth in the optimal measurement range while the data outside this range display noise which gets more pronounced for data further away from the optimal region. In other words, at low shear rates the torque exerted on the internal cylinder approaches its minimal measurable value and its measurement is increasingly subjected to error. The values recorded for shear rates lower than $60 \mathrm{~s}^{-1}$ are discarded from the graph as they may be considered random. For this reason, a wide range of shear rates is examined so that the rheological response of the tested fluid may be extrapolated for low shear rate values.

Although both test fluids applied in the gravity casting experiments may be generally considered Newtonian, their dynamic viscosity slightly increases with increasing shear rate. This behaviour may be well approximated with a linear function. When a linear regression analysis is applied to experimental data in Figure 7, the linear relation between the dynamic viscosity $\eta$ and the shear rate $\dot{\gamma}$ for the test fluid in the horizontal channel tests is $\eta=38.08+0.00278 \dot{\gamma}[\mathrm{mPa} \cdot \mathrm{s}]$. Obviously, the slope of the linear model is moderate.

In order to approximate the test fluid as Newtonian, the constant value of the dynamic viscosity can be determined e.g. as a median of average values from all performed tests. Averaging each test separately is crucial in order to gain equal statistical weight of every test, because several viscosity testing scenarios are applied. Based on the data from the range of shear rates in $\langle 200 ; 1945\rangle \mathrm{s}^{-1}$, i.e. within the applicable measurement range, the dynamic viscosity of the Newtonian model of the horizontal channel test fluid is $\eta=41.6 \mathrm{mPa} \cdot \mathrm{s}$ (shown as a red line in Figure 7). When considering moderate flow during the horizontal channel casting, this value might be slightly overestimated as an absolute measurement of the dynamic viscosity at extremely low shear rates is not possible.

The second test fluid used in the vertical labyrinth campaign displays qualitatively the same behaviour as the

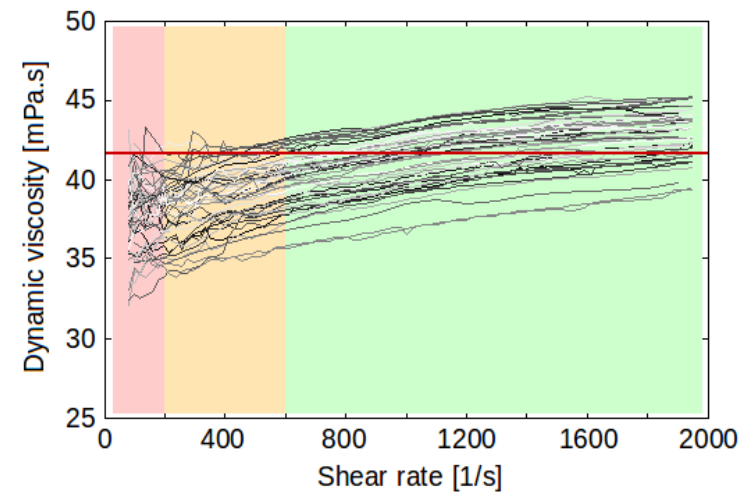

Fig. 7. Dynamic viscosity as a function of shear rate as recorded during all rheological measurements of the casting material during the horizontal channel campaign.

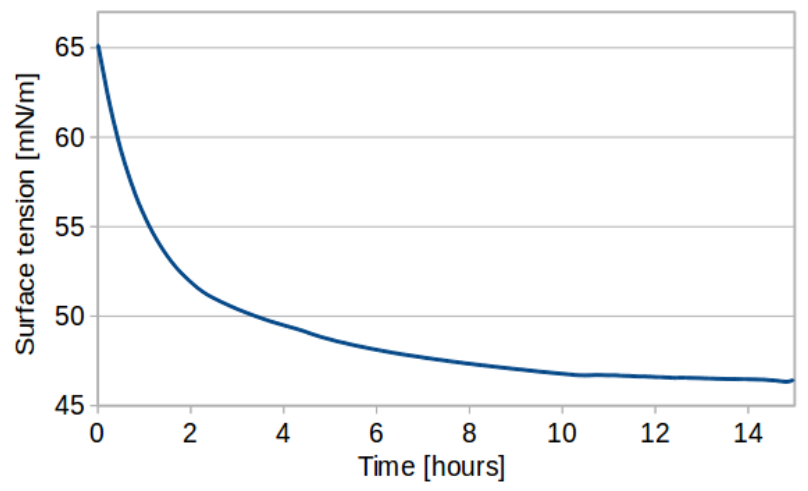

Fig. 8. Evolution of surface tension in time as recorded for a random specimen of the casting material used during the horizontal channel campaign.

test fluid used in the horizontal channel tests. Quantitatively, its dynamic viscosity is slightly higher. The median of average values from all viscosity measurements is $\eta=45.4 \mathrm{mPa} \cdot \mathrm{s}$ (based on the data for shear rates in $\left.\langle 200 ; 1945\rangle \mathrm{s}^{-1}\right)$. The linear function approximating the experimental data is $\eta=42.26+0.00279 \dot{\gamma}[\mathrm{mPa} \cdot \mathrm{s}]$. The slope of the linear function is about identical as in the case of the test fluid for the horizontal channel campaign.

In addition, a value of the surface tension is assessed for both test fluids. On the contrary to the dynamic viscosity measurements, the surface tension is examined for all used fluid samples after the gravity casting experiments and for only few fresh samples before selected gravity casting tests. For both the horizontal channel and the vertical labyrinth experimental campaigns, 25 surface tension measurements are executed. Two kinds of tests are performed, a standard Wilhelmy plate surface tension test and a continuous measurement of the forces acting (due to surface tension) on the Wilhelmy plate which is kept submerged at a constant depth.

Prior to the two sets of surface tension tests described above, long duration continuous Wilhelmy plate measurements of the test fluids were performed. These long term measurements reveil that the value of the surface tension 


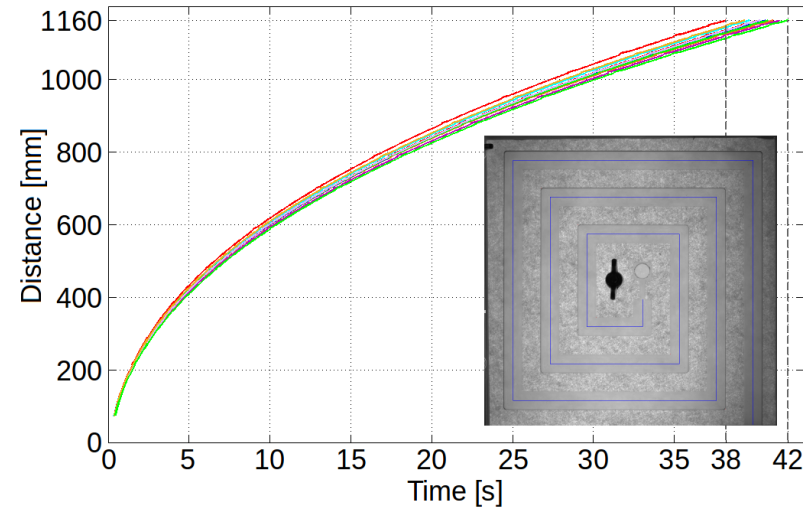

Fig. 9. Horizontal channel: propagation of the casting material along the channel centerline. The centerline of the horizontal channel is depicted as a blue line in the embedded photo.

changes in time and the test fluids behave as an aqueous surfactant solution, see Figure 8. In general, a high surface tension value can be recorded for the well stirred sample of the aqueous surfactant solution, but this value decreases as the sample gets settled and molecules of the surfactant become organised at the free surface. In a long time, the surface tension converges to a static value. In case of the used test fluids, the difference between the initial and the static value might become nearly $20 \mathrm{mN} / \mathrm{m}$ (the value has not fully converged during the 15 hour test).

Due to excessive time demands of the long term measurements, only the initial value of test fluid's surface tension is assessed for well stirred fresh samples as well as for samples used in the gravity casting experiments. Median of the initial surface tension of the fluid casted in the horizontal channel tests, vertical labyrinth tests respectively, is $\sigma=60 \mathrm{mN} / \mathrm{m}, \sigma=64 \mathrm{mN} / \mathrm{m}$ respectively.

In the end of each gravity casting campaign, the density measurements are performed by weighing a number of well defined sample volumes of test fluids (either fresh or used). The results show that both test fluids have the density of $\varrho=1.24 \mathrm{~g} / \mathrm{cm}^{3}$.

\subsection{Gravity casting}

The experimental setup is designed so that the entire execution of the casting tests can be well controlled. The most essential part of the experimental run is its initial phase. The gravity flow of the test fluid is initiated by a removal of the gate between the reservoir and the mold. In both experimental setups, the gate obstructs a $3 \mathrm{~mm}$ high horizontal channel which is a single inlet into the mold. During the gate removal, the gate slides vertically upwards which causes a gradual opening of the horizontal channel. The gate removal time is estimated based on camera images which are captured at $20 \mathrm{~Hz}$ framerate. Since a precise estimate is limited by the applied camera framerate, the gate removal time is considered to be less or equal to $50 \mathrm{~ms}$.

After the gate removal, the inlet channel is fully opened and the test fluid is free to flow into the mold under
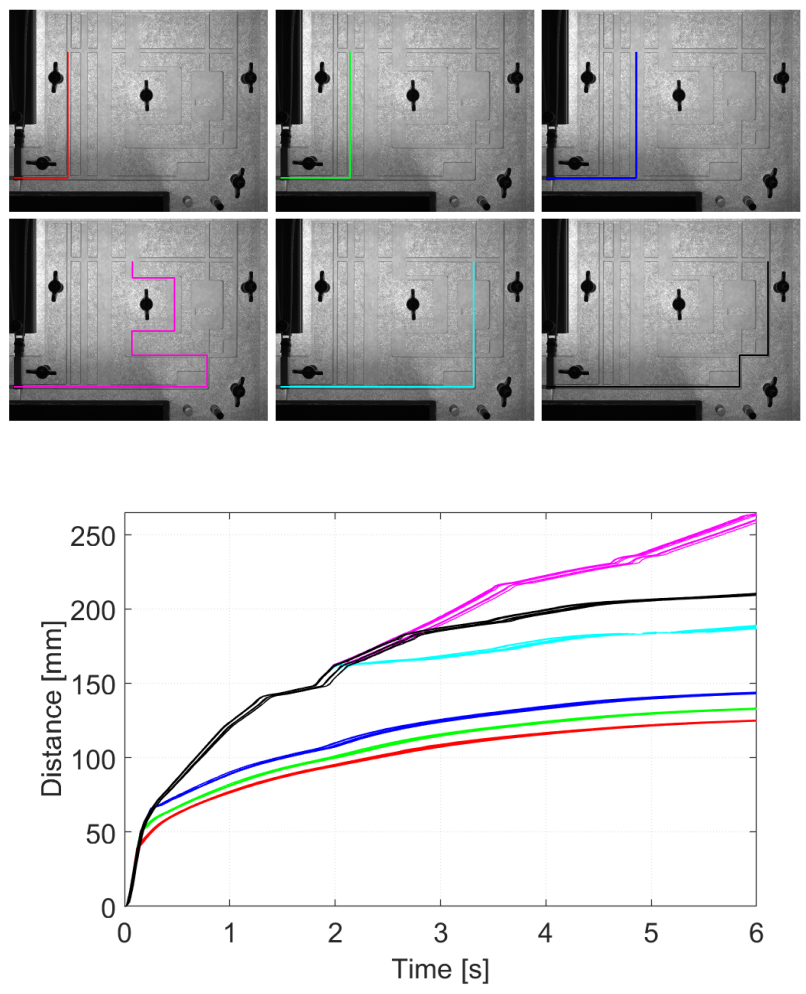

Fig. 10. Vertical labyrinth: propagation of the casting material along the six pathlines in the initial phase of the experiment. The selected pathlines in the labyrinth geometry are depicted in six different colours (top) and the propagation curves in the distancetime plot are colour-coded accordingly (bottom).

gravity. The most dramatic flow is observed right after the gate opening when the vertical reservoir is full of the casted test fluid. As the fluid level in the reservoir decreases, the gravity flow slows down. Nevertheless, due to the fact that the highly viscous test fluids are applied, the fluid flow inside both the horizontal channel and the vertical labyrinth can be considered laminar at all instants.

In all casting tests, a smooth convex shaped profile of the fluid front can be observed. This is apparent e.g. in Figure 6 (bottom right). A distinctive free surface profiles can be observed in channel corners, as shown in Figure 5 (bottom left) and Figure 5 (bottom right), and at labyrinth channel intersections.

Special attention is paid to the propagation of the casting material within the mold geometry. In order to gain quantitative results, a position of the fluid front along the channel centerline is examined in time (in terms of the distance from the wetted side of the gate).

Results of ten experimental runs of gravity casting into the horizontal channel geometry are shown in Figure 9. The plotted curves clearly indicate a decrease in propagation speed in time after a rapid initial phase of the gravity flow. Nevertheless, variation of about $10 \%$ can be observed in the arrival time to the very end of the $1160 \mathrm{~mm}$ long horizontal channel. This may be attributed to slight differences in the dam gate removal.

Within the vertical labyrinth, six pathlines are selected as depicted in Figure 10 (top). Propagation of the test 
fluid along these pathlines during the first 6 seconds after the gate removal is displayed in Figure 10 (bottom). Results of all ten tests are displayed, but the curves are coloured according to the pathline they refer to. Thus a fluid flow through the entire labyrinth can be easily identified. Since the propagation of the fluid through the horizontal channels is faster than in the vertical ones, the instants when the fluid starts moving vertically upwards or when it enters another horizontal channel are clearly marked. The red, green a blue curves that refer to the first three straight vertical channels display minimal variation in the results among indiviual tests performed. This is related to the very short distance the fluid travels from the gate. The variation in resulting fluid propagation is more pronounced for the longer pathlines, i.e. for the trident of vertical channels of various shapes. This is obvious especially in the longest pathline (coloured in magenta) which combines several horizontal and vertical channels into an S-like shape. Nevertheless, variations in Figure 10 (which shows first $250 \mathrm{~mm}$ of the path) are not as dramatic as in the case of significantly longer horizontal channel.

\section{Conclusions}

The presented experimental measurements of the gravity casting flow cover both the kinematics of the fluid propagation in the mold geometry and assessment of the test fluid physical properties. As a result, an extensive set of well controlled experimental data is recorded. The study aims at identification of a benchmark test and creation of a large dataset that could be used for validation of computational software tools, such as in [5].

This study is supported by the grant project GA $18-25734 \mathrm{~S}$ "Experimental and computational modelling of free surface flow of non-Newtonian fluids with dispersed particles" of the Czech Science Foundation and by the project LO1506 of the Czech Ministry of Education, Youth and Sports under the program NPU I.

\section{References}

[1] B. Sirrell, M. Holliday, J. Campbell, JOM, 48, 20-23 (1996).

[2] H. Zhao, I. Ohnaka, J. Zhu, Appl. Math. Model., 32, 185-194 (2008).

[3] J. Ha, P. Cleary, V. Ahuja, T. Nguyen, Proc. 2nd International Conference on CFD in the Minerals and Process Industries, CSIRO, Melbourne, Australia, 423428 (1999).

[4] T.A. Kowalewski, A. Cybulski, T. Sobiecki, Computational Methods and Experimental Measurements X, WIT Transactions on Modelling and Simulation, 30, 179-188 (2001)

[5] J. Vimmr, L. Lobovský, O. Bublík, T. Mandys, Proc. ECCM-ECFD 2018, Glasgow, United Kingdom (2018). http://www.eccmecfd2018.org/admin/files/filePaper/p1719.pdf 